\title{
Glueball spectrum from a potential model
}

\author{
Wei-Shu Hou \\ Department of Physics, National Taiwan University, Taipei, Taiwan 10764, Republic of China \\ Gwo-Guang Wong \\ Department of International Trade, Lan Yang Institute of Technology, Toucheng, Ilan, Taiwan 26141, Republic of China
}

(Received 28 July 2002; published 7 February 2003)

\begin{abstract}
The spectrum of two-gluon glueballs below $3 \mathrm{GeV}$ is investigated in a potential model with a dynamical gluon mass using the variational method. The short-distance potential is approximated by one-gluon exchange, while the long-distance part is taken as a breakable string. The mass and size of the radial as well as orbital excitations up to a principle quantum number $n=3$ are evaluated. The predicted mass ratios are compared with experimental candidates and lattice results.
\end{abstract}

DOI: 10.1103/PhysRevD.67.034003

PACS number(s): 12.39.Mk

Quantum chromodynamics (QCD) is widely accepted as the theory of strong interactions. It is generally believed that the gluon self-coupling in QCD implies the existence of bound states of confined gluons known as glueballs. The experimental discovery of these glueballs would be very important and would give further support to the theory of QCD. However, numerous technical difficulties have so far hampered our unequivocal identification of glueballs by experiment, largely because glueball states can mix strongly with nearby $q \bar{q}$ resonances. Nevertheless, the estimation of mass and size of pure gluon glueball states should still be pursued. This could guide experimental searches, as well as provide a calibration for models of glueballs.

Over the past 20 years there has been an ongoing effort to obtain a nonperturbative form for the gluon propagator. Perhaps one of the most interesting results is that the gluon may have a dynamically generated mass [1]. The existence of a mass scale, or the absence of a pole at $k^{2}=0$, is natural if one assumes that gluons do not propagate to infinity; i.e., these propagators describe confined gluons. The concept of a massive gluon has been widely used in independent field theoretic studies, and examples about the consequences of massive gluons can be found in the literature [2-7].

In this paper, we focus on the calculation of two-gluon glueball systems and extend our previous work [8] on the estimation of the mass and size of low-lying glueball states, using the variational method in the potential model of Cornwall and Soni $[9,10]$. The main feature of the present work is the consideration of radial as well as orbital excitations, up to principle quantum number $n=3$.

To exhibit both asymptotic freedom and the non-Abelian nature of QCD, gluon dynamics can be described as massive spin-1 fields interacting through one-gluon exchange and a breakable string. At short-distance the effective coupling constant of the gluon-gluon interaction becomes small and the interaction can be treated perturbatively. The short distance potential is approximated by one-gluon exchange and can be extracted from the tree-level Feynman amplitude of Fig. 1,

$$
V(\mathbf{r})=\int \frac{d^{3} q}{(2 \pi)^{3}} \frac{i e^{i \mathbf{q} \cdot \mathbf{r}}}{4 \sqrt{E_{1 f} E_{2 f} E_{1 i} E_{2 i}}} i \mathcal{M}_{f i},
$$

where $\mathbf{q}$ is the momentum transfer of the system. At long distance, the non-Abelian nature of QCD implies gluon confinement via nonperturbative effects. These nonperturbative effects are implemented by introducing a string potential $V_{\text {str }}$ which is assumed to be spin-independent,

$$
V_{\mathrm{str}}=2 m\left(1-e^{-\beta m r}\right) \text {, }
$$

where $\beta$ is related to the adjoint string tension $K_{A}$ via

$$
\beta=\frac{K_{A}}{2 m^{2}} .
$$

In the potential $V_{\text {str }}$, the color screening of gluons is brought about by a breakable string; that is, the adjoint string breaks when sufficient energy has been stored in it to materialize a gluon pair. This form of the string potential simulates the intergluonic potential as seen in lattice calculations [11].

Thus the gluon-gluon potential relevant for two-gluon glueballs is [8]

$$
\begin{aligned}
V_{2 g}(r)= & -\lambda\left\{\left[\frac{1}{4}+\frac{1}{3} \mathbf{S}^{2}+\frac{3}{2 m^{2}}(\mathbf{L} \cdot \mathbf{S}) \frac{1}{r} \frac{\partial}{\partial r}\right.\right. \\
& \left.-\frac{1}{2 m^{2}}\left((\mathbf{S} \cdot \nabla)^{2}-\frac{1}{3} \mathbf{S}^{2} \nabla^{2}\right)\right] \frac{e^{-m r}}{r} \\
& \left.+\left(1-\frac{5}{6} \mathbf{S}^{2}\right) \frac{\pi}{m^{2}} \delta^{3}(\mathbf{r})\right\}+2 m\left(1-e^{-\beta m r}\right),
\end{aligned}
$$

where $\lambda$ is defined as

$$
\lambda \equiv \frac{3 g^{2}}{4 \pi},
$$

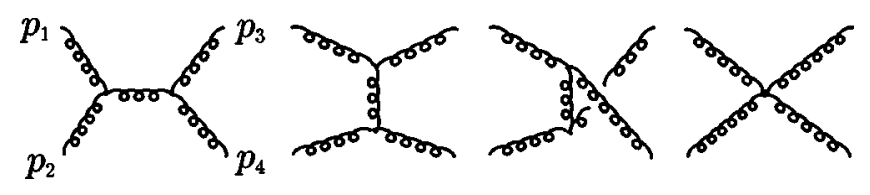

FIG. 1. Diagrams for $g g \rightarrow g g$ scattering. 
and is related to the strong-coupling strength of the process. The terms containing $\lambda$ are from the short-distance potential. Note that the color wave functions have been contracted out with the structure constants in gluon vertices [8], so that the strength of the gluon-gluon coupling is three times as large as that of the gluon-quark coupling. In Eq. (4), $\mathbf{S} \equiv \mathbf{S}_{1}+\mathbf{S}_{2}$ is the total spin of the two-gluon glueball. Note also that $\mathbf{S}_{1}$ acts on the polarization vectors $\mathbf{e}_{1}$ and $\mathbf{e}_{3}$ while $\mathbf{S}_{2}$ acts on the polarization vectors $\mathbf{e}_{2}$ and $\mathbf{e}_{4}$. Each spin operator $\mathbf{S}_{n}$ $=\left(S^{1}, S^{2}, S^{3}\right)$ is defined as $\left(S^{k}\right)_{i j}=-i \epsilon^{i j k}$, which satisfy

$$
\left[S^{i}, S^{j}\right]=i \epsilon^{i j k} S^{k},
$$

i.e., $S^{1}, S^{2}$, and $S^{3}$ are SU(2) group generators as desired.

For this gluon-gluon potential we are left with three parameters: effective gluon mass $m$, string breaking parameter $\beta$, and adjoint strong-coupling constant $\lambda$. In this model, the constituent gluon mass is evaluated to be $600-700 \mathrm{MeV}$, which is roughly twice the constituent quark mass [8]. In the intermediate or at least at long distances, the fundamental string tension $K_{F}$ is one of the most fundamental physical quantities in quark confinement, and is related to the Regge slope $\alpha^{\prime}$ by [12]

$$
K_{F}=\frac{1}{2 \pi \alpha^{\prime}}=0.18 \mathrm{GeV}^{2},
$$

with the experimental value for $\alpha^{\prime} \simeq 0.9 \mathrm{GeV}^{-2}$. The adjoint string tension $K_{A}$ for gluon confinement can be related by the strong evidence of the Casimir scaling hypothesis [13] on the lattice via

$$
\frac{K_{A}}{K_{F}} \approx \frac{9}{4}
$$

Thus, the string breaking parameter $\beta$ is about $0.4-0.6$. For the gluon propagator with a dynamical mass, the adjoint strong-coupling constant $\lambda$ at the one-loop level turns out to be [7]

$$
\lambda\left(Q^{2}\right)=\frac{36 \pi}{\left(33-2 n_{f}\right) \ln \left[\left(Q^{2}+\xi m^{2}\right) / \Lambda^{2}\right]},
$$

where $\xi \approx 4$. The higher-order correction at the two-loop level does affect the value, as shown in the case of the fundamental strong-coupling constant $\alpha_{\mathrm{s}}\left(Q^{2}\right)$ [14]. However, it has not been solved so far in the literature. Nevertheless, $\lambda$ is expected to be in the range of $1.5 \pm 0.5$.

The invariance of charge conjugation and parity for strong interactions implies that a two-gluon glueball must have quantum numbers $J^{\mathrm{PC}}=J^{(-1)^{L}(+)}$. On the other hand, since the gluon is a spin 1, color octet boson, the color singlet wave function of a two-gluon glueball is symmetric. Hence, a symmetric spin wave function with spin 0 or 2 must be accompanied by a symmetric spatial wave function with an even value of orbital angular momentum, and an antisymmetric spin wave function with spin 1 must be accompanied by an antisymmetric spatial wave function with an odd value of orbital angular momentum. In this way, one can count the number of states. For example, we have two $S$-wave, three $P$-wave, and six $D$-wave states with definite $J^{\mathrm{PC}}$ corresponding to principle quantum number $n=3$.

The Hamiltonian of a two-gluon glueball system is

$$
H=2 m-\frac{1}{m} \nabla^{2}+V_{2 g}
$$

Since the color wave function has been contracted out, the remaining wave function is of the form

$$
\begin{aligned}
\Psi_{n l j m}(\vec{r}) & \equiv|n l j m\rangle \propto \psi_{n l}(r)|j m\rangle \\
& \equiv \psi_{n l}(r) \sum_{m=m_{l}+m_{s}}\left\langle l m_{l} s m_{s} \mid j m\right\rangle Y_{l m_{l}}(\theta, \phi) \chi_{s m_{s}},
\end{aligned}
$$

where

$$
\chi_{s m_{s}}=\sum_{\lambda_{1} \lambda_{2}}\left\langle 1 \lambda_{1} 1 \lambda_{2} \mid s m_{s}\right\rangle \mathbf{e}_{1}^{\left(\lambda_{1}\right)} \mathbf{e}_{2}^{\left(\lambda_{2}\right)} .
$$

The trial radial wave fuctions $\psi_{n l}(r)$ are constructed by orthogonality as follows:

$$
\begin{gathered}
\psi_{10}(r) \propto e^{-a^{2} m^{2} r^{2}}, \\
\psi_{20}(r) \propto\left(1-a^{2} m^{2} r^{2}\right) e^{-a^{2} m^{2} r^{2} / 2}, \\
\psi_{21}(r) \propto a^{2} m^{2} r^{2} e^{-a^{2} m^{2} r^{2} / 2}, \\
\psi_{30}(r) \propto\left(1-\frac{62}{51} a^{2} m^{2} r^{2}+\frac{13}{68} a^{4} m^{4} r^{4}\right) e^{-a^{2} m^{2} r^{2} / 4}, \\
\psi_{31}(r) \propto a^{2} m^{2} r^{2}\left(1-\frac{3}{14} a^{2} m^{2} r^{2}\right) e^{-a^{2} m^{2} r^{2} / 4}, \\
\psi_{32}(r) \propto a^{4} m^{4} r^{4} e^{-a^{2} m^{2} r^{2} / 4},
\end{gathered}
$$

where $a$ is the variational parameter. The spin wave function $\chi_{s m_{s}}$ is constructed by the direct product of two-gluon polarization vectors $\mathbf{e}_{1}^{\left(\lambda_{1}\right)}$ and $\mathbf{e}_{2}^{\left(\lambda_{2}\right)}$ and, in turn, the total angular momentum eigenstate $|j m\rangle$ can be constructed by the direct product of an orbital eigenstate, the spherical harmonics $Y_{l m_{l}}(\theta, \phi)$, and the spin wave function $\chi_{s m_{s}}$. The coefficients $\left\langle 1 \lambda_{1} 1 \lambda_{2} \mid s m_{s}\right\rangle$ and $\left\langle l m_{l} s m_{s} \mid j m\right\rangle$ in Eqs. (11) and (12) are just the Clebsch-Gordan coefficients.

When considering the case with $L=0$ [8], we drop the spin-orbit and tensor terms in Eqs. (4). However, they do contribute for the case with $L>0$. Since only the tensor term in Eq. (4) is related to the total angular momentum eigenstates $|j m\rangle$, we need to calculate their expectation values. Defining the tensor operator as

$$
\mathbf{T}=\mathbf{S}^{2}-3(\mathbf{S} \cdot \hat{\mathbf{r}})^{2}
$$

integrating out the spherical harmonics, and doing spin algebraic calculation on spin wave functions, we obtain 


$$
\langle 00|\mathbf{T}| 00\rangle=0 \quad \text { for } \quad l=0, s=0
$$

and

$$
\langle 2 m|\mathbf{T}| 2 \quad m\rangle=0 \quad \text { for } \quad l=0, s=2 .
$$

For $l=1$ and $s=1$, we have

$$
\begin{aligned}
\langle 2 m|\mathbf{T}| 2 m\rangle & =\frac{1}{5}, \\
\langle 1 m|\mathbf{T}| 1 m\rangle & =-1, \\
\langle 00|\mathbf{T}| 00\rangle & =2,
\end{aligned}
$$

for $l=2$ and $s=0$, we have

$$
\langle 2 m|\mathbf{T}| 2 m\rangle=0 ;
$$

and for $l=2$ and $s=2$, we have

$$
\begin{gathered}
\langle 4 m|\mathbf{T}| 4 m\rangle=\frac{12}{7} \\
\langle 3 m|\mathbf{T}| 3 m\rangle=-\frac{24}{7} \\
\langle 2 m|\mathbf{T}| 2 m\rangle=-\frac{9}{7} \\
\langle 1 m|\mathbf{T}| 1 m\rangle=3 \\
\langle 0 \quad 0|\mathbf{T}| 00\rangle=6
\end{gathered}
$$

Hence the contribution to the glueball mass $M$ can be written as

$$
M / m=2+E_{K}+E_{Y}+E_{\delta}+E_{L S}+E_{T}+E_{s t r},
$$

where

$$
\begin{gathered}
E_{K}=-\frac{1}{m^{2}}\left\langle n l\left|\nabla^{2}\right| n l\right\rangle, \\
E_{Y}=-\frac{\lambda}{m}\left[\frac{1}{4}+\frac{1}{3} S(S+1)\right]\left\langle n l\left|\frac{e^{-m r}}{r}\right| n l\right\rangle, \\
E_{\delta}=-\frac{\lambda \pi}{m^{3}}\left[1-\frac{5}{6} S(S+1)\right]\left\langle n l\left|\delta^{3}(\mathbf{r})\right| n l\right\rangle, \\
E_{L S}=\frac{3 \lambda}{2 m^{3}} \mathbf{L} \cdot \mathbf{S}\left\langle n l\left|\left(\frac{1}{r^{2}}+\frac{m}{r}\right) \frac{e^{-m r}}{r}\right| n l\right\rangle, \\
E_{T}=-\frac{\lambda}{2 m^{3}}\langle j m|\mathbf{T}| j m\rangle\left\langle n l\left|\left(\frac{1}{r^{2}}+\frac{m}{r}+\frac{m^{2}}{3}\right) \frac{e^{-m r}}{r}\right| n l\right\rangle, \\
E_{\mathrm{str}}=2\left\langle n l\left|\left(1-e^{-\beta m r}\right)\right| n l\right\rangle,
\end{gathered}
$$

and $|n l\rangle \equiv \psi_{n l}(r)$.
We then use the variational method with trial radial wave function $\psi_{n l}(r)$ to evaluate the glueball mass $M$ and rootmean-square radius $r_{\text {rms }}=\sqrt{\left\langle r^{2}\right\rangle}$ for each glueball state. The lightest scalar and tensor glueballs have been investigated in Ref. [8]. For the lightest scalar glueball there is an attractive $\delta$-function term in the potential and hence the Hamiltonian is unbounded from below. It has been conjectured that this maximum attraction channel in the $0^{++}$state could be related to the gluon condensation that triggers confinement. We proposed a physical solution by smearing the gluon fields; that is, we replace the $\delta$ function by the smearing function

$$
D(r)=\frac{k^{3} m^{3}}{\pi \frac{3}{2}} e^{-k^{2} m^{2} r^{2}},
$$

which approaches $\delta^{3}(\mathbf{r})$ for $k \rightarrow \infty$. In contrast to the lightest scalar glueball, the lightest tensor glueball is stable since the $\delta$-function term is repulsive. As the variation is slight in the mass and size estimation [8] of a glueball in the $\lambda-\beta$ parameter space, we take the central values of $\lambda=1.5$, and $\beta$ $=0.5[15]$.

The lattice result of $M\left(1{ }^{1} S_{0}\right), M\left(1{ }^{5} S_{2}\right)=1730,2400$ $\mathrm{MeV}$ is taken as input for the lightest $0^{++}$and $2^{++}$glueballs, respectively $[16,18]$. From the $2^{++}$input, we find the constituent gluon mass $\sim 670 \mathrm{MeV}$, about twice the constituent quark mass, and the lightest tensor glueball is found to have the typical hadron size of $\sim 0.8 \mathrm{fm}$.

The value for $k$ in Eq. (22) is fixed by the mass ratio

$$
\frac{M\left(1{ }^{5} S_{2}\right)}{M\left(1{ }^{1} S_{0}\right)} \cong 1.39
$$

from the converging lattice and experimental results. The size of the lightest scalar glueball is found to be a mere $\sim 0.1 \mathrm{fm}$. We note that, although we always have an attractive $\delta$-function term in the potential for scalars, only for the lightest scalar glueball is the smearing of gluon fields needed. For all other scalars, the $\delta$-function potential gives very small mass corrections. One can consider the sum of kinetic energy and $\delta$-function terms as the effective kinetic energy. We find that the sum contributes less than $12 \%$ to all glueball masses, except for the lightest scalar glueball, which is $43 \%$. This may be marginal for a nonrelativistic treatment, and stretching the applicability of our relativistic expansion.

We list the mass spectrum and size of two-gluon glueballs up to $n=3$ in Table I.

From Table I, we see that the glueball mass $M$ decreases with increasing orbital angular momentum $L$ for fixed $n$. For fixed $n$ and $L=0,1(2), M$ increases (decreases) with total spin angular momentum $S$. In turn, both $M$ and size increase with total angular momentum $J$ for fixed $n, L$, and $S$. On the other hand, $M$ increases with $n$ at fixed $L$ and $S$. All these increments are slight, except for the $n=1$ case in which the attractive $\delta$ potential is present.

Our calculations show that glueball masses are almost independent of $\lambda$, and increase with $\beta$ only slightly [15]. Al- 
TABLE I. Masses and sizes of two-gluon glueballs up to $n$ $=3$, with the lightest $0^{++}$and $2^{++}$masses taken as input.

\begin{tabular}{|c|c|c|c|c|c|}
\hline$n$ & $L$ & $S$ & $J^{\mathrm{PC}}$ & $M(\mathrm{MeV})$ & $r_{\mathrm{rms}}(\mathrm{fm})$ \\
\hline & & 0 & $0^{++}$ & $\{1730\}$ & 0.1 \\
\hline \multirow[t]{3}{*}{1} & 0 & 2 & $2^{++}$ & $\{2400\}$ & 0.8 \\
\hline & & 0 & $0^{++}$ & 2710 & 2.0 \\
\hline & 0 & 2 & $2^{++}$ & 2730 & 1.9 \\
\hline \multirow[t]{8}{*}{2} & & & $0^{-+}$ & 2570 & 0.7 \\
\hline & 1 & 1 & $1^{-+}$ & 2605 & 1.0 \\
\hline & & & $2^{-+}$ & 2615 & 1.1 \\
\hline & & 0 & $0^{++}$ & 2790 & 2.6 \\
\hline & 0 & 2 & $2^{++}$ & 2810 & 2.4 \\
\hline & & & $0^{-+}$ & 2765 & 2.4 \\
\hline & 1 & 1 & $1^{-+}$ & 2770 & 2.4 \\
\hline & & & $2^{-+}$ & 2775 & 2.4 \\
\hline \multirow[t]{6}{*}{3} & 2 & 0 & $2^{++}$ & 2700 & 1.6 \\
\hline & & & $0^{++}$ & 2685 & 1.1 \\
\hline & & & $1^{++}$ & 2690 & 1.3 \\
\hline & 2 & 2 & $2^{++}$ & 2693 & 1.5 \\
\hline & & & $3^{++}$ & 2694 & 1.6 \\
\hline & & & $4^{++}$ & 2695 & 1.7 \\
\hline
\end{tabular}

though our pure gluon glueballs are different from real glueball states which can mix strongly with nearby $q \bar{q}$ resonances, the better way to compare with experimental data is to take mass ratios, either to eliminate the $\lambda$ and $\beta$ dependence or to reduce the effect of mixing. We list in Table II the glueball masses in increasing order, together with their corresponding mass ratios with respect to the lightest tensor glueball. Comparison with Table I shows that, for a given quantum number, the ordering in mass is also the ordering in size.

Although our predictions rely on the inputs of the lightest scalar and tensor glueball masses, 1730 and $2400 \mathrm{MeV}$, respectively, from lattice calculation, we find that our predicted mass ratios do find experimental correspondence, sometimes even better than comparing with lattice calculations. This may be due to the rather straightforward physical picture of a potential model. However, the experimental situation is far from settled, and comparison with the lattice is quite necessary.

As stated, the lightest tensor glueball is much more stable within the model than the lightest scalar glueball, hence all mass ratios are normalized to this state. We find that, except for the peculiar lightest $0^{++}$state, all ratios of two-gluon glueball masses are of order 1 , and mass ratios of three-gluon to two-gluon glueballs are of order $3 / 2$, respectively. This is consistent with a constituent picture. We then need to understand the difference from the lattice spectrum, which suggests a considerably higher excitation energy.

Going back to Table I, we note that the constituent picture gives certain multiplet structures governed mostly by $n$ and $L$, with relatively small splittings in $S$ (except for $n=1$ ). We note that there are spin-orbit and tensor forces at work. Thus, the "first excitation" from the $n=1$ states seems to be the $(n, L, S)=(2,1,1)[$ rather than $(2,0,0)]$ states of $0^{-+}, 1^{-+}$, and $2^{-+}$. The masses are all around $2600 \mathrm{MeV}$, or $\sim 1.08$ times the lightest $2^{++}$. They are also of similar size to the lightest $2^{++}$, the $0^{-+}$actually smaller, hence they are likely more relevant in production processes.

The $0^{-+}$may be most interesting since it is the lightest pseudoscalar glueball. Its mass of $2570 \mathrm{MeV}$ is in remarkable agreement with the lattice result of $2590 \mathrm{MeV}$. The heaviness suggests [21] that there may be little glueball mixture in $\eta$ and $\eta^{\prime}$, and Okubo-Zweig-Iizuka (OZI) rule violation in these mesons arises from vacuum effects. The $1^{-+}$ state with exotic quantum numbers cannot be a $q \bar{q}$ meson and is also very interesting. Again, its heaviness compared to hybrid candidates such as $\hat{\rho}(1600)$ [22] suggests that the search for the lightest hybrids is not complicated by the presence of glueballs. For the $2^{-+}$state, the mass of $2615 \mathrm{MeV}$ in the constituent model is in contrast with the lattice result of $3100 \mathrm{MeV}$, which has a $500 \mathrm{MeV}$ excitation energy with respect to the lowest-lying $0^{-+}$state. This large gap is absent in our constituent model, which can largely be traced to the tensor force.

The $(n, L)=(2,0)$ and $(3,0)$ states of $0^{++}$and $2^{++}$are straightforward radial excitations of the $n=1$ states. Since the lightest $0^{++}$is especially light, the excitation energy is more than $900 \mathrm{MeV}$. But for the $2^{++}$state, it seems that the radial excitation energy is only $300 \mathrm{MeV}$ or so, while going on to $n=3$ states, the excitation energy is less than $100 \mathrm{MeV}$. We notice that the size has also reached beyond $2 \mathrm{fm}$, hence the drop in excitation energy reflects the approach to string breaking beyond a couple of Fermis. Such behavior, however, is not seen on the lattice, where the second $2^{++}$state is also almost $900 \mathrm{MeV}$ higher than the lightest one, which again appears more "stringy" ("Regge").

The $(n, L, S)=(3,2,0)$ and $(3,2,2)$ states in the constituent model are close to degenerate, which arise again due to a balance between spin-orbit and tensor forces. Interestingly, the $0^{++}$state constructed out of $L=S=2$, possible only starting with $n=3$, is slightly lighter than the radial excitation of the lightest $0^{++}$state. It is thus actually the second lightest such state, with a size of $1.1 \mathrm{fm}$, comparable to the lightest $2^{++}$state but only half that of the radially excited $0^{++}$state. We note that its mass of $2685 \mathrm{MeV}$ is in excellent agreement with the second $0^{++}$state from the lattice, although our radial excitation states are also not in disagreement. The $(n, L, S)=(3,2,0) 2^{++}$state is also slightly lighter and smaller in size than the radially excited $2^{++}$state. The interpretation of excitation energy for $0^{++}$versus $2^{++}$ therefore could be rather different between our model and the lattice calculations.

The above two special $L=2$ states are accompanied by the host of $1^{++}, 2^{++}, 3^{++}$, and $4^{++}$glueballs, which are comparable in mass and gradually growing in size. They could be more interesting than the "usual" radial excitations of the lightest $0^{++}$and $2^{++}$states, or $0^{-+}, 1^{-+}$, and $2^{-+}$ states, which are two-and-a-half Fermi in size and rather large. In contrast, the lattice $3^{++}$and $4^{++}$states are close to $3700 \mathrm{MeV}$, again appearing as stringy excitations.

The experimental results are rather uncertain at present, but there does seem to be many states in the $2000 \mathrm{MeV}$ 
TABLE II. Comparison of predicted glueball masses (mass ratios, normalized to lightest $2^{++}$) with lattice [16] and sample experimental data. The superscripts $a, b, c$, and $d$ indicate data coming from [17], [18], [19], and [20], respectively. The experimental numbers are not meant to match the states listed to the left. The last three entries with $3 g$ in front of the $J^{\mathrm{PC}}$ are for three-gluon glueballs [8]. The lightest $0^{++}$and $2^{++}$masses are taken as input. All masses are in $\mathrm{MeV}$ units.

\begin{tabular}{|c|c|c|c|}
\hline$J^{\mathrm{PC}}$ & Constituent & Lattice & Experiment \\
\hline & $\{1730\}(0.72)$ & $1730(0.72)$ & $1500^{b}(0.76)$ \\
\hline \multirow[t]{5}{*}{$0^{++}$} & $2685(1.12)$ & $2670(1.11)$ & $2105^{b}(1.06)$ \\
\hline & $2710(1.13)$ & & $2320^{c}(1.17)$ \\
\hline & $2790(1.16)$ & & \\
\hline & $\{2400\}(1.00)$ & $2400(1.00)$ & $1980^{b}(1.00)$ \\
\hline & $2693(1.12)$ & $3290(1.37)$ & $2020^{d}(1.02)$ \\
\hline \multirow[t]{3}{*}{$2^{++}$} & $2700(1.13)$ & & $2240^{d}(1.13)$ \\
\hline & $2730(1.14)$ & & $2370^{d}(1.20)$ \\
\hline & $2810(1.17)$ & & \\
\hline \multirow[t]{2}{*}{$0^{-+}$} & $2570(1.07)$ & $2590(1.08)$ & $2140^{d}(1.08)$ \\
\hline & $2765(1.15)$ & & $2190^{b}(1.11)$ \\
\hline \multirow[t]{2}{*}{$1^{-+}$} & 2605 (1.09) & & \\
\hline & $2770(1.15)$ & & \\
\hline \multirow[t]{2}{*}{$2^{-+}$} & 2615 (1.09) & $3100(1.29)$ & $2040^{d}(1.03)$ \\
\hline & $2775(1.16)$ & $3890(1.62)$ & $2300^{d}(1.16)$ \\
\hline $1^{++}$ & $2690(1.12)$ & & $2340^{d}(1.18)$ \\
\hline \multirow[t]{2}{*}{$3^{++}$} & $2694(1.12)$ & $3690(1.54)$ & $2000^{d}(1.01)$ \\
\hline & & & $2280^{d}(1.15)$ \\
\hline \multirow[t]{2}{*}{$4^{++}$} & $2695(1.12)$ & $3650^{a}(1.52)$ & $2044^{d}(1.03)$ \\
\hline & & & $2320^{d}(1.17)$ \\
\hline $3 g\left(0^{-+}\right)$ & 3780(1.58) & $3640(1.52)$ & \\
\hline $3 g\left(1^{--}\right)$ & $3680(1.53)$ & $3850(1.60)$ & \\
\hline $3 g\left(3^{--}\right)$ & $3690(1.54)$ & $4130(1.72)$ & \\
\hline
\end{tabular}

region as compared to lattice suggestions. In part for reasons of comparison, we take here $1980 \mathrm{MeV}$ for a $2^{++}$candidate mass [18]. Glueball candidates abound but they are very hard to pin down. In contrast to lattice results, especially for the glueballs with size less than $2 \mathrm{fm}$, the richness of our spectrum should give some hope for experimental search, although one clearly has a long way to go. For the lattice calculations, it is not clear to us whether the short-distance spin-orbit and tensor interactions, of great importance to our model (though mysteriously balancing each other), are replicated.

In our model, the size of the lightest scalar glueball is of order $0.1 \mathrm{fm}$. The extreme smallness may be an artifact of the treatment of the attractive $\delta$ potential, but we do expect a physically smaller lightest scalar from the heuristic point of view, because of the extra attraction. A more direct calculation of the $0^{++}$glueball mass and size on the lattice would require relatively fine lattice spacings [23]. It would be interesting to see if our result of small $0^{++}$size could be borne out on the lattice. The lightest pseudoscalar glueball mass is $2570 \mathrm{MeV}$. However, there are the other two $0^{-+}$states with mass 2765 and $3780 \mathrm{MeV}$ composed of two and three gluons, respectively. Mixing among them should make the lightest $0^{-+}$two-gluon glueball mass lighter. On the other hand, despite the lattice QCD prediction that the lowest-lying $1^{-+}$ glueball has mass heavier than $J / \psi[24]$, one should not exclude the $1^{-+}$glueball in a search below $3 \mathrm{GeV}$ [25]. The $1^{--}$and $3^{--}$glueballs only exist in three-gluon glueball states, hence their masses should be heavier than $3 \mathrm{GeV}$. Scaling from the lattice result of $M_{2++}=2000-2400 \mathrm{MeV}$ $[16,26,27]$, the mass range of these glueballs is $3.1-3.7 \mathrm{GeV}$, right in the ballpark of $J / \psi$ and $\psi^{\prime}$ masses. The proximity of the $1^{--}$glueball to $J / \psi$ or perhaps $\psi^{\prime}$ may be what is needed from comparison of $J / \psi$ and $\psi^{\prime}$ two-body hadronic decays [28]. Before closing, we remark that the masses of the purely gluonic low-lying states $0^{++}, 2^{++}$, and $0^{-+}$have been reported to be around 1.5, 2.0, and $2.05 \mathrm{GeV}$ [29], respectively, by using QCD spectral sum rules (QSSR). They are suitably close to the experimental candidates, and possibly mixed with $q \bar{q}$ resonances. Nevertheless, it is interesting that all glueball masses in our model would become lighter if we take the QSSR result of $M\left(1{ }^{1} S_{0}\right), M\left(1{ }^{5} S_{2}\right)$ $=1.5,2.0 \mathrm{GeV}$ as input for the lightest $0^{++}$and $2^{++}$glueballs.

In conclusion, two-gluon glueballs have been studied in a potential model with constituent gluons. The potential is approximated by one-gluon exchange plus a breakable string. The mass and size of radial and orbital excitations, up to principle quantum number $n=3$, are evaluated by the variational method. With the lowest-lying $0^{++}$and $2^{++}$masses from the lattice taken as input, all masses of two-gluon glueballs are found to be below $3 \mathrm{GeV}$ with size less than $3 \mathrm{fm}$. The predicted masses for the second $0^{++}$state as well as the lowest $0^{-+}$state are in excellent agreement with the lattice. Further excitation patterns, however, differ considerably. While lattice calculations find fewer states populating the $3000 \mathrm{MeV}$ range, hence an excitation energy of 500-900 $\mathrm{MeV}$, our constituent model gives two more $0^{++}$states, four more $2^{++}$states, and an additional $0^{-+}$state, which are 12-17\% heavier than the lightest tensor. This pattern may well be more consistent with experiment. There are also a number of $1^{\mp+}, 2^{-+}, 3^{++}$, and $4^{++}$glueballs. Besides a rather small size for the lightest scalar, there is one $0^{++}$, $2^{++}, 0^{-+}, 1^{-+}$, and $2^{-+}$glueball each that is $1 \mathrm{fm}$ in size, each one the lightest member for the given quantum number, with $1^{++}$only slightly larger. The number of glueballs clustering at $13 \%$ or heavier than the lightest $2^{++}$glueball are typically $2 \mathrm{fm}$ in size. Mass and size seem positively correlated. While the model certainly has its limitations, its heuristic nature may provide some help in the long quest for uncovering glueball states in Nature.

This work is supported in part by the National Science Council of R.O.C. under Grant No. NSC-90-2112-M-002022 . 
[1] J. M. Cornwall, Phys. Rev. D 26, 1453 (1982).

[2] G. Parisi and R. Petronzio, Phys. Lett. 94B, 51 (1980).

[3] F. Halzen, G. I. Krein, and A. A. Natale, Phys. Rev. D 47, 295 (1993); M. B. Gay Ducati, F. Halzen, and A. A. Natale, ibid. 48, 2324 (1993); J. R. Cudell and B. U. Nguyen, Nucl. Phys. B420, 669 (1994).

[4] J. H. Field, Int. J. Mod. Phys. A 9, 3283 (1994); J. P. Liu and W. Wetzel, hep-ph/9611250.

[5] M. Consoli and J. H. Field, Phys. Rev. D 49, 1293 (1994); J. Phys. G 23, 41 (1997).

[6] J. R. Forshaw, J. Papavassiliou, and C. Parrinello, Phys. Rev. D 59, 074008 (1999).

[7] A. Mihara and A. A. Natale, Phys. Lett. B 482, 378 (2000).

[8] W. S. Hou, C. S. Luo, and G. G. Wong, Phys. Rev. D 64, 014028 (2001).

[9] J. M. Cornwall and A. Soni, Phys. Lett. 120B, 431 (1983).

[10] W. S. Hou and A. Soni, Phys. Rev. D 29, 101 (1984).

[11] C. Bernard, Phys. Lett. 108B, 431 (1982); Nucl. Phys. B219, 341 (1983).

[12] P. Goddard, J. Goldston, C. Rebbi, and C. B. Thorn, Nucl. Phys. B56, 109 (1973); K. Johnson and C. B. Thorn, Phys. Rev. D 13, 1934 (1976).

[13] G. S. Bali, Nucl. Phys. B (Proc. Suppl.) 83, 422 (2000).

[14] K. G. Chetyrkin, J. H. Kühn, and M. Stenhauser, Comput. Phys. Commun. 133, 43 (2000).
[15] In general, $\lambda$ dependence can be neglected, and the larger the $\beta$, the heavier the glueball.

[16] C. J. Morningstar and M. J. Peardon, Phys. Rev. D 60, 034509 (1999).

[17] D. Q. Liu and J. M. Wu, Mod. Phys. Lett. A 17, 1419 (2002).

[18] D. V. Bugg, M. J. Peardon, and B. S. Zou, Phys. Lett. B 486, 49 (2000).

[19] A. V. Anisovich et al., Phys. Lett. B 471, 271 (1999); Nucl. Phys. A662, 319 (2000).

[20] B. S. Zou, Nucl. Phys. A655, 41 (1999).

[21] W. S. Hou and C. Y. Ko, hep-ph/9708314.

[22] P. R. Page, Phys. Lett. B 415, 205 (1997).

[23] C. Morningstar and M. J. Peardon, Nucl. Phys. B (Proc. Suppl.) 83, 887 (2000).

[24] G. Bali et al., Phys. Lett. B 309, 378 (1993).

[25] D. M. Li, H. Yu, and Q. X. Shen, Commun. Theor. Phys. 35, 44 (2001).

[26] A. Vaccarino and D. Weingarten, Phys. Rev. D 60, 114501 (1999).

[27] C. Liu, Nucl. Phys. B (Proc. Suppl.) 94, 255 (2001); J. Sexton, A. Vaccarino, and D. Weingarten, Phys. Rev. Lett. 75, 4563 (1995); G. S. Bali et al., Phys. Lett. B 309, 378 (1993).

[28] M. Suzuki, hep-ph/0006296; Phys. Rev. D 65, 097507 (2002).

[29] S. Narison, Nucl. Phys. A675, 54c (2000). 